\title{
Loading working memory enhances affective priming
}

\author{
MARK ROTTEVEEL and R. HANS PHAF \\ University of Amsterdam, Amsterdam, The Netherlands
}

\begin{abstract}
Stronger affectivepriming (Murphy \& Zajonc, 1993) with suboptimal (i.e., reduced consciousness) than with optimal (i.e., full consciousness) prime presentation suggests that nonconscious processes form an important part of emotions. Merikle and Joordens (1997) have argued that both impoverished presentation and divided attention can produce suboptimal conditions and result in parallel effects. We manipulated attention by means of a concurrent working memory load while keeping presentation duration constant, as participants evaluatedJapanese ideographs that were preceded by happy, neutral, or angry faces (affective priming) and male or female faces (nonaffective priming). In contrast to nonaffective priming, affective priming was larger with divided attention than with focused attention. It is concluded that manipulations of stimulus quality and of attention can both be used to probe the distinction between conscious and nonconscious processes and that the highest chances of obtaining the pattern of stronger priming with suboptimal presentation than with optimal presentation occur in the affective domain.
\end{abstract}

To investigate what it means whether or not a stimulus is processed consciously, Baars and McGovern (1993; see also Merikle, 1992) proposed the general method of experimentally manipulating level of consciousness and contrasting the effects of conscious (optimal) and less conscious (suboptimal) conditions (i.e., a contrastive analysis). This method can be applied to the study of many independent variables and has revealed a number of qualitative differences (e.g., Cheesman \& Merikle, 1984; Greenwald, 1992; Groeger, 1984, 1988; Jacoby \& Whitehouse, 1989) which suggest that conscious and nonconscious processes do not always have the same form. In addition, the finding of stronger effects in less conscious than in fully conscious conditions contradicts the view that suboptimal processing constitutes only diluted conscious processing (i.e., with some trials inadvertently processed consciously). This type of result has primarily been found in the affective domain (Bornstein, 1989; Fulcher \& Hammerl, 2001; Janssen, Everaerd, Spiering, \& Janssen, 2000; Murphy \& Zajonc, 1993; Rotteveel, de Groot, Geutskens, \& Phaf, 2001; Stapel, Koomen, \& Ruys, 2002), whereas weaker suboptimal effects than optimal effects are usually obtained in other, more nonaffective, domains (e.g., Lubke, Kerssens, Phaf, \& Sebel, 1999; Maxfield, 1997; Murphy \& Zajonc, 1993, Experiments 3-6; but see De Fockert, Rees, Frith, \& Lavie,

The authors are grateful to Julie Boland, Phil Merikle, and an anonymous reviewer for very helpful comments. We thank Susanne de Boer and Marisa Stoffers for their assistance in performing the experiments. R.H.P. is in the Department of Psychonomics; M.R. is in the Department of Psychonomics and the Department of Social Psychology. Correspondence about this paper should be sent to M. Rotteveel, Department of Social Psychology, University of Amsterdam, Roetersstraat 15, 1018 WB Amsterdam, The Netherlands (e-mail: (mrotteveel@uva.nl).
2001). Merikle and Joordens (1997) have argued that divided attention and impoverished (i.e., short, masked) presentation can both be used to produce suboptimal conditions, so that both should have corresponding effects. To our knowledge, however, this correspondence has not been explicitly studied in the affective domain. We have investigated whether stronger suboptimal affective priming than optimal affective priming (Murphy \& Zajonc, 1993) also occurs when suboptimal conditions are achieved by dividing attention instead of through impoverished presentation and whether this contrasts with nonaffective priming.

Murphy and Zajonc (1993) investigated priming in affective and nonaffective evaluation of unfamiliar Chinese ideographs. Affectively valenced (i.e., happy or angry faces) and control (polygons, faces with neutral expressions) stimuli were presented in either a suboptimal (4 msec, or about $10 \mathrm{msec}$ effectively; see Winkielman, Zajonc, \& Schwarz, 1997), or an optimal (1,000 msec) fashion by means of a projection tachistoscope. Because the absence of an effect in a (direct) task measuring conscious effects cannot guarantee that all conscious processing has been exhaustively excluded (Cheesman \& Merikle, 1984), the cautious terminology of suboptimal and optimal, instead of subliminal and supraliminal, presentation was adopted by Murphy and Zajonc. In the first two experiments, happy and angry faces led to reliable, valence-congruent, shifts in ideograph evaluation only with suboptimal prime presentation. The stronger suboptimal as opposed to optimal results preclude the possibility that suboptimal effects are caused only by conscious processing "leaking through." Nonaffective priming of size (Experiment 3), symmetry (Experiment 4), and gender (Experiment 5), however, revealed congruent shifts only in optimal conditions. 
Rotteveel et al. (2001) have argued that the finding of stronger suboptimal as opposed to optimal affective priming may be rather brittle and sensitive to problems associated with impoverished prime presentation. Eye-blinks or saccades may, for instance, cause participants to miss suboptimal primes. Because one is effectively blind during a saccade (Morgan, 1994; Tovée, 1996), a brief stimulus presented during the saccade cannot exert a priming effect. The stronger suboptimal as opposed to optimal affective priming might thus be weakened only with short presentation due to the influence of saccades. Single frame presentation on a computer screen might also dilute suboptimal effects. The picture is built up pixel by pixel and line by line. The visibility of a single pixel corresponds to the effective persistence of the specific phosphor used in the screen (about $4 \mathrm{msec}$, Bridgeman, 1998). This time is generally shorter than a single screen frame, and prime presentation may sometimes be too short to allow for any processing at all. Impoverished presentation may, furthermore, allow for a confounding of presentation condition and instruction. Murphy and Zajonc (1993) instructed participants to ignore the faces only in optimal conditions, but did not tell them about the suboptimal primes. The participants might have applied the controlled strategy of counteracting the prime influences only in optimal conditions, whereas in suboptimal conditions no effort was spent to discard possible effects of the faces. Although Rotteveel et al. (2001) also obtained stronger suboptimal as opposed to optimal priming with impoverished presentation when instructions were equated, it seems better to have some form of suboptimal presentation that allows more fully for the application of the same instruction.

Merikle and Joordens (1997) proposed that manipulations of attention and of stimulus quality (i.e., in terms of presentation duration and masking) have parallel effects. They obtained equivalent effects of both manipulations with false recognition, exclusion failure, and a variant of the Stroop-task. To achieve suboptimal processing, attention was diverted away from a stimulus that was presented as long as it was in the optimal conditions. Monitoring a sequence of auditory digits for three consecutive odd digits served to divide attention. Merikle and Joordens argued that finding similar qualitative differences supports the view that both manipulations address the same underlying process distinction (i.e., of conscious and nonconscious processing). In terms of a contrastive analysis, both manipulations may thus serve to reduce level of consciousness.

Merikle and Joordens's (1997) position is strengthened further if the stronger suboptimal as opposed to optimal priming is also reproduced with attentional manipulations. Affective priming seems to be most suited, because the pattern appears to be predominant in the affective domain (but see Clore \& Ortony, 2000). A nonaffective priming task was, however, also included in order to investigate further whether affective value was indeed the critical factor in the priming pattern. We manipulated at- tention in both an affective priming (i.e., with emotional faces as primes) and nonaffective priming (i.e., male and female faces serving as primes) task. Attention was varied by concurrently loading working memory (see Mulligan \& Stone, 1999). Participants were instructed to maintain, and to reproduce at the end of the trial, a digitletter string of seven elements with divided attention, or an empty string (i.e., a "-") with focused attention. The participants were instructed emphatically to perform the concurrent working memory task as accurately as possible. They also had to judge intuitively whether Japanese ideographs (Rotteveel et al., 2001) preceded by the faces represented something either positive or negative (i.e., affective task), or something masculine or feminine (i.e., nonaffective task). We expected stronger affective priming and weaker nonaffective priming under conditions of divided as opposed to focused attention.

\section{METHOD}

\section{Participants}

A total of 96 (65 female) 1 st-year psychology students $(M=$ 21.96 years old, $S D=2.69$ ) from the University of Amsterdam participated in the two tasks for course credit. All were right-handed and had normal or corrected-to-normal vision. The experiment was announced as a test of "intuitive knowledge of the Japanese language." Only participants naive with regard to eastern languages were enrolled. The participants were divided into two groups of 48 for the affective and nonaffective priming tasks, respectively.

\section{Design}

The affective task had a 2 (focused vs. divided attention) $\times 3$ (happy, neutral, or angry face) mixed factorial design, and the nonaffective task had a 2 (focused vs. divided attention) $\times 2$ (male vs. female face) mixed factorial design. Attention was manipulated between participants. The coupling of prime to target was counterbalanced over participants. Order of presentation was randomized by the computer for each participant individually. Affective and gender ratings of the ideographs served as dependent variables. As an exploratory dependent variable, the proportion of correct reproductions of individual string elements was calculated per condition for each participant.

\section{Material and Apparatus}

Forty-eight pictures (in grayscale, measuring $12.5 \mathrm{~cm} \times 15.8 \mathrm{~cm}$ on the screen) of Caucasian models ( 24 female) with happy, neutral, and angry facial expressions were selected from the Ekman and Friesen (1976) and Matsumoto and Ekman (1988) picture sets. Also 24 male and 24 female faces with a neutral expression (transformed to the same format as that of the affective pictures) served as primes (Lundqvist, Flykt, \& Öhman, 1998). In pilot experiments, the selected faces received the most consistent ratings on their respective attributes. The 48 prime faces for the nonaffective task were moreover judged affectively neutral in these experiments. Forty-eight different Japanese ideographs of equal complexity (i.e., consisting of 10-12 black strokes on a light background) served as targets. Forty-eight different digit-letter strings consisting of seven elements (e.g., 3D6F8G2) were prepared for the divided attention conditions. The strings consisted of digits (1-9) and letters (B, C, D, F, $\mathrm{G}, \mathrm{H}, \mathrm{J}, \mathrm{K}, \mathrm{L})$ combined according to three rules: every string started with a digit, digits and letters alternated, and digits and letters were not repeated within a string.

Participants made two-alternative forced-choice ratings with their index finger on marked keys from the keyboard. The labels (the 
Table 1

Average Verbal Working-Memory Span (With Standard Deviations) in the 15-Word Test for the Participant Groups in the Focused and Divided Attention Conditions of the Affective and Nonaffective Priming Conditions

\begin{tabular}{llllll}
\hline \multirow{2}{*}{ Priming } & \multicolumn{2}{l}{ Focused Attention } & & \multicolumn{2}{c}{ Divided Attention } \\
\cline { 2 - 5 } & Avg. & $S D$ & & Avg. & $S D$ \\
\hline Affective & 4.96 & 0.86 & 4.96 & 0.55 \\
Nonaffective & 5.20 & 0.72 & 4.88 & 0.68 \\
\hline
\end{tabular}

ideograph represented something "negative" or "positive," or something "masculine" or "feminine") were balanced over both keys across participants. The experiment was controlled by a Power Macintosh 4400/200 computer equipped with a 15-in. screen. Viewing distance was approximately $60 \mathrm{~cm}$, resulting in an $11.8^{\circ}$ horizontal and $14.8^{\circ}$ vertical visual angle. The fixation point was presented for $500 \mathrm{msec}$ and was followed by a blank field for $500 \mathrm{msec}$. The digitletter string (or "-," with zero load) appeared for $3.5 \mathrm{sec}$ and was followed by a blank field for $1 \mathrm{sec}$. The second fixation point was presented for $0.5 \mathrm{sec}$ and was immediately followed by the face for $1 \mathrm{sec}$, after which the target was presented for $1 \mathrm{sec}$. If a string was presented before the trial, participants typed on the keyboard what they remembered of the string after rating the ideograph.

\section{Procedure}

To determine individual working memory capacity, a modified Dutch version of the 15-words test (Deelman, 1990) was first administered. The participants were instructed to reproduce lists of Dutch words spoken by the experimenter. List length started with 2 words, and each time two lists had been presented, the list length increased by 1 additional word, until a length of 7 words was reached. Whenever participants made a mistake in one of the two lists with the same number of words, the preceding number of words was taken as the verbal working memory span.

The participants were instructed that the experiment was about intuitive knowledge of the meaning of signs from an unfamiliar language. They were informed that this knowledge presumably surfaces best when they are actively engaged in another task. The importance of the concurrent working memory task was stressed, and the participants were encouraged to keep trying although they would probably not succeed in reproducing all strings correctly. They were also told to ignore the faces appearing before the ideographs, which served to increase the concurrent load in other experimental conditions. The participants first practiced on the rating task for 10 trials. After 24 experimental trials, a 2-min break was given. The experiment was concluded by an exit interview.

\section{RESULTS}

Generally, the participants reported they had been well aware of the faces before the ideographs, even with divided attention. Working memory load was relatively homogeneous with divided attention throughout the two tasks. The average scores on the 15 -words test (see Table 1) did not differ significantly among the four groups. In the divided attention conditions, the affective primes did not clearly influence string reproduction (positive, $0.52, S D=0.16$; neutral, $0.53, S D=0.12$; negative, $0.55, S D=0.13$ ), nor did the gender primes in the nonaffective task (male, 0.61, $S D=0.16$; female, $0.63, S D=0.17$ ).

The affective and nonaffective ratings (see Tables $2 \mathrm{~A}$ and $2 \mathrm{~B}$ ) were first subjected to a combined analysis. To have the same number of levels of the factor prime in the affective and the nonaffective tasks, the neutral condition in the former task was omitted. Because we are primarily interested in differential priming patterns between divided and focused attention conditions, the actual coding of the judgments does not matter. We choose to code both negative and masculine ratings with a " 1 ," and positive and feminine ratings with a " 2 ." In the 2 (affective vs. nonaffective task) $\times 2$ (divided vs. focused attention $) \times($ prime " 1 " vs. prime " 2 ") ANOVA, only the type of prime constituted a within-participants factor. If stronger suboptimal as opposed to optimal priming occurred only in affective conditions, a threeway interaction of task, attention, and prime would be expected. This interaction was indeed found to be significant $[F(1,92)=7.03, p<.01]$. In addition, an overall priming effect $[F(1,92)=27.0, p<.01)$ and a task $\times$ prime interaction $[F(1,92)=7.25, p<.01]$ were obtained. Prime " 1 " $(1.45, S D=0.16)$ and prime " 2 " $(1.57, S D=$ $0.16)$ resulted overall in congruent ideograph ratings. The priming effect was, moreover, somewhat larger in the affective (negative, $1.43, S D=0.18$; positive, $1.62, S D=$ 0.15 ) than in the nonaffective task (male, $1.46, S D=0.14$; female, $1.52, S D=0.14$ ). No further main effects or interactions were significant. To see whether the effect of dividing attention indeed diverged in the affective and nonaffective tasks, the two tasks were analyzed separately.

\section{Affective Priming}

Ideographs were overall evaluated congruently with the affective primes (positive, 1.62, $S D=0.15$; neutral, $1.52, S D=0.14$; negative, $1.43, S D=0.18$ ). Because the affective ratings of the happy, neutral, and angry faces in the pilot experiment obeyed a linear relationship, a linear trend could also be expected in the priming effects. The overall linear trend of affective valence $[F(1,92)=24.2$, $p<.01]$ was indeed significant and was qualified by an interaction with attention condition $[F(1,92)=5.47, p<$ $.05]$, indicating that the priming effect was stronger with divided than with focused attention (see Table 2A). No significant quadratic trends were observed $(F \mathrm{~s}<1)$. Planned comparisons revealed that with divided attention, priming by positive faces differed reliably from priming by negative faces [paired, one-tailed $t(23)=5.79$, $p<.01]$, whereas this difference did not reach the level of significance with focused attention $[t(23)=1.66, p=$

Table 2A

Means (With Standard Deviations) of the Affective Ratings $(1=$ Negative, $2=$ Positive $)$ as a Function of Attention and of Facial Expression

\begin{tabular}{cccccc}
\hline & \multicolumn{2}{c}{$\begin{array}{c}\text { Focused } \\
\text { Attention }\end{array}$} & & \multicolumn{2}{c}{$\begin{array}{c}\text { Divided } \\
\text { Attention }\end{array}$} \\
\cline { 2 - 3 } \cline { 5 - 6 } Facial & $M$ & $S D$ & & $M$ & $S D$ \\
\hline Positive & 1.56 & 0.17 & & 1.69 & 0.11 \\
Neutral & 1.52 & 0.16 & & 1.54 & 0.11 \\
Negative & 1.46 & 0.18 & & 1.39 & 0.18 \\
\hline
\end{tabular}


Table 2B

Means (With Standard Deviations) of the Nonaffective Ratings (1 = Masculine, 2 = Feminine $)$ as a Function of Attention and of Model Gender

\begin{tabular}{lccccc}
\hline & \multicolumn{2}{c}{$\begin{array}{c}\text { Focused } \\
\text { Attention }\end{array}$} & & \multicolumn{2}{c}{$\begin{array}{c}\text { Divided } \\
\text { Attention }\end{array}$} \\
\cline { 2 - 3 } \cline { 5 - 6 } Gender & $M$ & $S D$ & & $M$ & $S D$ \\
\hline Male & 1.42 & 0.12 & & 1.50 & 0.17 \\
Female & 1.52 & 0.12 & & 1.52 & 0.17 \\
\hline
\end{tabular}

.0553]. Affective priming was not qualified by participant gender in any respect. In the divided attention condition, moreover, there was no significant relation $(r=$ .27 , n.s.) between string performance and affective priming (i.e., the ideograph rating difference with positive and negative primes).

\section{Nonaffective Priming}

In view of the expectations, a priori tests were performed. With focused attention, the priming effect (see Table 2B) was significant [paired, one-tailed $t(23)=3.19$, $p<.01]$, but it disappeared with divided attention $[t(23)=$ 0.467 , n.s.). Participant gender again did not influence the priming effects. With divided attention, moreover, the strength of priming again did not depend on string performance $(r=.19$, n.s.).

\section{DISCUSSION}

Stronger affective priming occurred with divided than with focused attention, but nonaffective conditions showed the more classical pattern of weaker priming with divided attention. The notion that divided attention and impoverished presentation have parallel effects (Merikle \& Joordens, 1997) is, therefore, supported by the finding of stronger suboptimal as opposed to optimal affective priming and of weaker suboptimal as opposed to optimal nonaffective priming with both types of manipulations. The finding of parallel effects by Merikle and Joordens and by us clearly suggests that the same type of conscious processing is affected by impoverished presentation and by dividing attention. Moreover, these results imply that predominantly nonconscious processes can be studied even under conditions in which participants subjectively report that they are aware of the primes. The results therefore support the notion that no direct measure can be used to exhaustively exclude all conscious processing (Cheesman \& Merikle, 1984; Merikle, 1992) and that a contrastive analysis is the better research strategy for characterizing conscious and nonconscious processes.

Dividing attention for obtaining suboptimal presentation not only has practical advantages, but also has theoretical implications. Alternative interpretations in terms of timing of stimuli (e.g., habituation processes) can be excluded because of the matching of presentation durations. With divided attention, moreover, less conscious percep- tion may be excluded than with impoverished presentation. The participants generally reported that they had seen the face preceding the ideograph even when working memory was loaded. This remaining consciousness with divided attention presents a problem only when one wants to exhaustively exclude all consciousness and wants to show that all remaining processing must be nonconscious (an indirect-without-direct effect; Merikle, 1992). Creating suboptimal conditions by dividing attention thus makes sense only in a contrastive analysis and not if one wants to obtain an indirect-without-directeffect. The generalization of these effects to other consciousness manipulations further strengthens the idea that a fundamental contrast is involved and that the distinction between conscious and nonconscious processes is meaningful.

Clore and Ortony (2000) have attacked the notion that stronger suboptimal as opposed to optimal priming is specific to emotion. They argue that it should be explained in the same way as other types of priming, and claim that "The only difference is that the visual mask, which ensures that the image is available for only a few milliseconds, interferes with the episodic knowledge of having seen the stimulus. But it does not interfere with the semantic knowledge of what was seen" (p.43). This fails to explain, however, why in similar conditions the opposite pattern (i.e., weaker suboptimal as opposed to optimal priming; Murphy \& Zajonc, 1993, Experiments 3-6) should occur with nonaffective priming. Moreover, the participants in our experiment appeared well able to report the presence of faces despite the working memory load. Episodic knowledge was thus not suppressed by divided attention. If anything, moreover, dividing attention actually reduces semantic processing (e.g., Craik, Govoni, Naveh-Benjamin, \& Anderson, 1996; Mulligan \& Stone, 1999). When, for instance, participants searched a prime word for a specific letter and attention was redirected to nonsemantic stimulus attributes, semantic priming was reduced or it even disappeared (Maxfield, 1997). If affective priming is equivalent to semantic priming, we should therefore have obtained less, instead of more, affective priming with divided attention.

The stronger suboptimal as opposed to optimal priming does not seem restricted only to the affective domain. De Fockert et al. (2001) recently reported stronger suboptimal interference than optimal interference by (nonemotional) faces in a name classification task. A working memory load resulted in increased face-related activity in the visual cortex, owing to presentation of the distractor faces and to increased interference effects. De Fockert et al. argued that working memory is a prerequisite for directing attention to relevant stimuli and for ignoring irrelevant stimuli. To also explain our results, however, the idea that divided attention decreased conscious control (i.e., to ignore the faces), so that nonconscious response tendencies became more dominant, needs to be supplemented with the hypothesis that such response tendencies are generally stronger for affective than for nonaf- 
fective stimuli. Because affective processes may be biologically (and genetically) prepared (Öhman, 1986; Öhman \& Mineka, 2001), there is a higher chance of finding such nonconscioustendencies in the affective domain than in other domains. This does not preclude, however, the occurrence of stronger suboptimal than optimal priming outside affective processing (e.g., owing to the highly automatized tendency to recognize faces; De Fockert et al., 2001). Even with affective processing, moreover, the inhibition of nonconscious processing may result from learning and our extensive training to exclude emotions from conscious reasoning.

The present account of affective priming (for a more detailed connectionist model, see Den Dulk, Capalbo, \& Phaf, in press) still allows for some attentional modulation of emotional face processing, even at the lowest levels. Pessoa, McKenna, Gutierrez, and Ungerleider (2002) obtained evidence in an fMRI study for top-down influences of attention, even at the level of the amygdala. At first sight, this would seem at odds with our finding of increased affective priming with divided attention. The crucial question here, however, is how affective and nonaffective processing differ so that dividing attention reduces nonaffective priming and can even increase affective priming. This question can be answered in connectionistterms by assuming "prewired" (i.e., biologically prepared; see Öhman \& Mineka, 2001) connections that have a larger influence on the activations than does top-down attentional modulation. For nonaffective stimuli, similarly strong connections may be formed after frequent stimulus encounters, so that processing of the stimuli is automatized and less affected by attentional modulation.

Particularly in the affective domain, attention and sufficient free working memory capacity are required to keep nonconscious response tendencies under control. Impoverished presentation may have effects similar to those of divided attention, because the stimulus is not processed sufficiently to allow for attentional control (see also Klinger, 2001). Whether the nonconscious processes have an evolutionary origin (see Den Dulk, Heerebout, \& Phaf, 2003) or have been automatized in the individual's lifetime remains a question for further research. The more frequent occurrence of the stronger suboptimal as opposed to optimal pattern with affective than with nonaffective processing, however, appears to favor a biological preparation.

\section{REFERENCES}

BAARS, B. J., \& MCGOVERN, K. (1993). Does philosophy help or hinder scientific work on consciousness? Consciousness \& Cognition, 2, 1827.

BoRnstein, R. F. (1989). Exposure and affect: Overview and metaanalysis of research 1968-1987. Psychological Bulletin, 106, $265-$ 289.

BRIDGEMAN, B. (1998). Durations of stimuli displayed on video display terminals: $(n-1) / f+$ persistence. Psychological Science, 9, 232-233.

Cheesman, J., \& Merikle, P. M. (1984). Priming with and without awareness. Perception \& Psychophysics, 36, 387-395.

Clore, G. L., \& Ortony, A. (2000). Cognition in emotion: Always, sometimes, or never? In R. D. Lane, \& L. Nadel (Eds.), Cognitive neuroscience of emotion (pp. 24-61). New York: Oxford University Press.

Craik, F. I. M., Govoni, R., Naveh-Benjamin, M., \& Anderson, N. D. (1996). The effects of divided attention on encoding and retrieval processes in human memory. Journal of Experimental Psychology: General, 125, 159-180.

Deelman, B. G. (1990). Neuropsychologische diagnostiek [Neuropsychological diagnostics]. In F. Luteyn, B. G. Deelman, \& P. M. G. Emmelkamp (Eds.), Diagnostiek in de klinische psychologie (pp. 136161). Houten, The Netherlands: Bohn, Stafleu en van Loghum.

De Fockert, J. W., ReEs, G., Frith, C. D., \& Lavie, N. (2001). The role of working memory in visual selective attention. Science, 291, 18031806.

Den Dulk, P., Capalbo, M. \& Phaf, R. H. (in press). A connectionist dual-route model for affective priming. Cognitive Processing.

Den Dulk, P., Heerebout, B. T., \& Phaf, R. H. (2003). A computational study into the evolution of dual-route dynamics for affective processing. Journal of Cognitive Neuroscience, 15, 194-208.

EKMAN, P., \& Friesen, W. V. (1976). Pictures offacial affect. Palo Alto, CA: Consulting Psychologists Press.

FulCHER, E. P., \& HAMMERL, M. (2001). When all is revealed: A dissociation between evaluative learning and contingency awareness. Consciousness \& Cognition, 10, 524-549.

Greenwald, A. G. (1992). New look 3: Unconscious cognition reclaimed. American Psychologist, 47, 766-779.

GROEGER, J. A. (1984). Evidence of unconscious semantic processing from a forced-error situation. British Journal of Psychology, 75, 305314.

GroEGER, J. A. (1988). Qualitatively different effects of undetected and unidentified auditory primes. Quarterly Journal of Experimental Psychology, 40A, 323-339.

JACOBY, L. L., \& WhitehouSE, K. (1989). An illusion of memory: False recognition influenced by unconscious perception. Journal of Experimental Psychology: General, 118, 126-135.

Janssen, E., Everaerd, W., Spiering, M., \& Janssen, J. (2000). Automatic processes and the appraisal of sexual stimuli: Towards an information processing model of sexual arousal. Journal of Sex Research, 37, 8-23.

KLINGER, M. R. (2001). The roles of attention and awareness in the false recognition effect. American Journal of Psychology, 114, 93-114.

Lubke, G. H., Kerssens, C., Phaf, R. H., \& Sebel, P. S. (1999). Dependence of explicit and implicit memory on hypnotic state in trauma patients. Anesthesiology, 90, 670-680.

Lundqvist, D., Flykt, A., \& ÖHman, A. (1998). The Karolinska directed emotional faces. Stockholm: Karolinska Institutet.

MATSUMOTO, D., EKMAN, P. (1988). Japanese and Caucasianfacial expressions of emotion (JACFEE) [Slides]. San Francisco: University of California, San Francisco, Human Interaction Laboratory.

MAXFIELD, L. (1997). Attention and semantic priming: A review of prime task effects. Consciousness \& Cognition, 6, 204-218.

Merikle, P. M. (1992). Perception without awareness. American Psychologist, 47, 792-795.

Merikle, P. M., \& JoORdens, S. (1997). Parallels between perception without attention and perception without awareness. Consciousness \& Cognition, 6, 219-236.

Morgan, M. J. (1994). When it pays not to see. Nature, 371, 473.

Mulligan, N. W., \& STONE, M. (1999). Attention and conceptual priming: Limits on the effects of divided attention in the category-exemplar production task. Journal of Memory \& Language, 41, 253-280.

MURPHY, S. T., \& ZAJONC, R. B. (1993). Affect, cognition and consciousness: Affective priming with optimal and suboptimal stimulus exposures. Journal of Personality \& Social Psychology, 64, 723-739.

ÖHman, A. (1986). Face the beast and fear the face: Animal and social fears as prototypes for evolutionary analyses of emotion. Psychophysiology, 23, 123-145.

ÖHman, A., \& MineKA, S. (2001). Fears, phobias, and preparedness: Toward an evolved module of fear and fear learning. Psychological Review, 108, 483-522.

Pessoa, L., McKenna, M., Gutierrez, E., \& Ungerleider, L. G. 
(2002). Neural processing of emotional faces requires attention. Proceedings of the National Academy of Sciences, 99, 11458-11463.

Rotteveel, M., De Groot, P., Geutskens, A., \& Phaf, R. H. (2001). Stronger suboptimal than optimal affective priming? Emotion, 1, 348-364.

StAPEL, D. A., KoOmen, W., \& RuYs, K. I. (2002). The effects of diffuse and distinct affect. Journal of Personality \& Social Psychology, 83, 60-74.
TovéE, M. J. (1996). An introduction to the visual system. Cambridge: Cambridge University Press.

Winkielman, P., ZAJONC, R. B, \& SchWARZ, N. (1997). Subliminal affective priming resists attributional interventions. Cognition \& Emotion, 11, 433-465.

(Manuscript received June 5, 2002; revision accepted for publication April 24, 2003.) 